\title{
Exploring the biophysical properties of phytosterols in the plasma membrane for novel cancer prevention strategies
}

\author{
AUTHORS \\ Omar Fakiha, Didem Sanver, ${ }^{\mathrm{b}, \mathrm{c}}$, David Kane ${ }^{\mathrm{b}}$ and James L Thorne ${ }^{\mathrm{b}}$
}

AFFILIATIONS

a. School of Medicine, Faculty of Medicine and Health, Leeds Teaching Hospitals Trust, UK, LS9 7TF

b. School of Food Science and Nutrition, Faculty of Mathematics and Physical Sciences, University of Leeds, UK, LS2 9JT.

c. Department of Food Engineering, Necmettin Erbakan University, Koycegiz Kampusu, 420701 Konya, Turkey

\section{CORRESPONDING AUTHOR}

James L Thorne j.1.thorne@ leeds.ac.uk

\begin{abstract}
Cancer is a global problem with no sign that incidences are reducing. The great costs associated with curing cancer, through developing novel treatments and applying patented therapies, is an increasing burden to developed and developing nations alike. These financial and societal problems will be alleviated by research efforts into prevention, or treatments that utilise off-patent or repurposed agents.
\end{abstract}

Phytosterols are natural components of the diet found in an array of seeds, nuts and vegetables and have been added to several consumer food products for the management of cardio-vascular disease through their ability to lower LDL-cholesterol levels. In this review, we provide a connected view between the fields of structural biophysics and cellular and molecular biology to evaluate the growing evidence that phytosterols impair oncogenic pathways in a range of cancer types. The current state of understanding of how phytosterols alter the biophysical properties of plasma membrane is described, and the potential for phytosterols to be repurposed from cardio-vascular to oncology therapeutics. Through an overview of the types of biophysical and molecular biology experiments that have been performed to date, this review informs the reader of the molecular and biophysical mechanisms through which phytosterols could have anti-cancer properties via their interactions with the plasma cell membrane. We also outline emerging and under-explored areas such as computational modelling, improved biomimetic membranes and ex vivo tissue evaluation. Focus of future research in these areas should improve understanding, not just of phytosterols in cancer cell biology but also to give insights into the interaction between the plasma membrane and the genome. These fields are increasingly providing meaningful biological and clinical data but iterative experiments between molecular biology assays, biosynthetic membrane 
studies and computational membrane modelling improve and refine our understanding of the role of different sterol components of the plasma membrane.

\section{KEYWORDS}

Cancer chemoprevention; diet and nutrition; phytosterols; membrane biophysics; oncogenic signalling; lipid rafts.

\section{ABBREVIATIONS}

AFM - Atomic Force Microscopy;

$\mathrm{BCa}$ - Breast Cancer;

BCRP - Breast Cancer Resistance Protein;

BPH - Benign Prostate Hyperplasia;

BRASS - Brassicasterol;

CAMP - Campesterol;

CAV1 - Caveolin 1;

CHOL - Cholesterol;

CVD - Cardio-Vascular Disease;

DCIS - Ductal Carcinoma In-situ;

DMPC - 1,2-dimyristoyl-sn-glycero-3-phosphocholine;

DOPC - 1,2-dioleoyl-sn-glycero-3-phosphocholine;

DOX - doxorubicin ;

DPPC - 1,2-dipalmitoyl-sn-glycero-3-phosphocholine;

DSPC - 1,2-distearoyl-sn-glycero-3-phosphocholine;

egg SM - Sphingomyelin;

EMT - Epithelial to Mesenchymal Transition;

EPIC - European Prospective Investigation into Cancer and Nutrition;

ERGO - Ergosterol;

FFQ - Food Frequency Questionnaire; 
$\mathrm{L}_{\mathrm{o}}$ - Liquid Ordered Phase;

$\mathrm{L}_{\mathrm{d}}$ - Liquid Disordered Phase;

$\mathrm{L}_{\beta}$ - Liquid Gel Phase;

LDL - low-density lipoprotein;

LUVs - Large Unilamellar Vesicles;

LXR - Liver X Receptor;

MDR - Multi-Drug Resistance;

MRP1 - Multiple Resistance Protein 1;

mTOR - mammalian Target of Rapamycin;

NDNS - National Diet and Nutrition Survey;

NR - Nuclear Receptor;

PTF - paclitaxel;

PC - phosphocholine;

Pgp - P-glycoprotein;

POPC - 1-palmitoyl-2-oleoyl-sn-glycero-3-phosphocholine;

PSM - palmitoylsphingomyelin;

SAXS - Small Angle X-ray Scattering;

SIT - $\beta$-sitosterol;

TAM - Tamoxifen; 


\section{Cancer Prevention}

The financial cost of cancer treatment is a significant burden on societies around the world. The role diet plays in cancer incidence and prevention is unequivocal with the most recent estimates suggesting that diet is a highly modifiable risk factor in upwards of $10 \%$ of cancers [1]. It is increasingly apparent that government healthcare agencies do not have the resources to treat their way out of the crisis, meaning cancer prevention provides the only realistic solution. Cancer prevention falls into three distinct categories. Primary prevention describes strategies that "treat" the entire population, including the healthy who will not go on to develop cancer. The expectation is that a proportion of individuals who without intervention, would have gone on to develop cancer, will instead stay cancer free. This approach requires a substantially rare side-effect profile and low cost of administering the agent to a large population. Secondary prevention strategies attempt to stop cancer from developing in those who are known to be at risk as they have been diagnosed with a significant risk factor such as colon adenoma, benign prostate hyperplasia or ductal carcinoma in situ. A higher side-effect profile can be tolerated as the population faces a higher degree of risk of developing serious disease so the urgency for prevention is greater. Tertiary prevention is defined as successful treatment of cancer where local or distal relapse is prevented, either at the time of surgery, radiology or systemic chemo/endocrine-therapy or in an on going manner as exemplified by the use of Tamoxifen for several years after surgical resection. There are several examples of cancer chemoprevention agents, but as yet many still have side-effect profiles that restrict their use. Tamoxifen for example is highly efficacious at tertiary breast cancer $(\mathrm{BCa})$ prevention [2-4] but increases the risk of other reproductive system cancers [5]. Aspirin reduces primary colorectal cancer risk $[6,7]$ but healthcare professionals remain reluctant to prescribe it owing to the increased risk of gastro-intestinal side-effects in some populations [8,9]. Over-treatment is unavoidable in the primary prevention setting, so the key requirements are that any chemoprevention agent must have tolerable/acceptable side-effects, be cost-effective, readily available and effective. Ideally, the compounds would already be available in consumer products at concentrations required for therapeutic intervention. Dietary factors are therefore ideal candidates, to either be consumed by personalised tailoring of diet for those at risk, or efficacious compounds could be turned into standardised nutraceuticals, or integrated into the food chain through crop engineering or food additives thus providing mass protection.

\section{Phytosterols}

Phytosterols are plant-derived sterols, similar to cholesterol (CHOL), that play structural roles in both plant and animal cell membranes (Figure 1). In the human diet campesterol (CAMP), $\beta$-sitosterol (SIT) and stigmasterol (STIG) are the most abundant phytosterols and typically consumed at around 50200mg/day [10-12]. Phytosterols are distinct from CHOL in function in the lipid bilayer and are not precursors of mammalian steroid hormones, seco-steroids or bile acids, as is the case for CHOL. 
Phytosterols do however influence intra-cellular signalling that can both replicate or be antagonistic of CHOL metabolites. Dietary intake of phytosterols by humans can lower their blood CHOL by reducing intestinal absorption [13]. Phytosterol added food-products are thus recommended to individuals at risk of cardio-vascular disease (CVD) but who are unable to tolerate statins, the preferred pharmacological intervention for CHOL management.

It is an important note for this review that phytosterols are unlikely to act on cellular systems purely through the plasma membrane. Their structural similarity to cholesterol oxidation products (oxysterols) means they are considered selective modulators of the Liver X Receptor (LXR) [14] and they act as inhibitors of other transcription factors such as FXR [15]. LXR has been implicated in BCa growth and spread [16], but also in repression of melanoma [17] and prostate cancer [18], underlining the disease and site contextual nature of its genomic actions. Further exploration of phytosterols as selective modulators of the LXR pathway is required to separate the membrane biophysical roles in cancer cell biology from their genomic actions.

\subsection{Dietary phytosterols and health}

For the clinical management of elevated cholesterol, plant sterols and stanols have been used to reduce circulating LDL-C concentrations in a dose dependent manner. A daily dietary intake of between $2-3 \mathrm{~g}$ of phytosterols achieves the maximal lowering of LDL-C levels at around 10-12\% [19] and is recommended by the US National Cholesterol Education Program as a life-style modification [20]. A recent analysis of the National Diet and Nutrition Survey (NDNS) revealed that less than $0.1 \%$ of a representative UK population is likely to be consuming this level of dietary phytosterols, and few if any of those actively trying to attain CHOL lowering through dietary methods achieve such high intakes (K. Nicoll Baines personal communication). Furthermore, data from the European Prospective Investigation into Cancer and Nutrition (EPIC) cohort indicates that dietary phytosterol intake is as low as 0.3g/day in a non-selected UK [21], and high-CVD risk Spanish [22] and Dutch [23] populations. Whilst CHOL absorption from the diet is typically greater than 50\% of that consumed [24], uptake of phytosterols is substantially lower but is not insignificant with approximately $0.5-3 \%$ of dietary phytosterols being absorbed [25]. Dietary assessments combined with mass-spectrometry quantification of plasma phytosterols concentrations [22], indicate that $0.3 \mathrm{~g} /$ day of dietary phytosterols typically translates to serum levels in the low micro molar range, but circulating concentrations have been reported as high as 100uM in plasma in older studies [26]. More recent and far larger studies [27] have measured serum phytosterol concentrations that are in agreement with the EPIC studies indicating circulating phytosterols in the general population are in the low micro molar range (SIT ca. 5uM, CAMP ca. $7 \mathrm{uM}$, STIG ca. $0.25 \mathrm{uM}$ ). 


\subsection{Phytosterols and cancer}

The loss of sensitivity to extra- and intra-cellular metabolic, apoptotic and anti-proliferative cues, as well as an increased sensitivity to growth signals are key stepping-stones to oncogenic progression [28]. These cellular changes, induced either by oncogene activation, loss of tumour suppressor function, epigenetic disruption or sustained signalling from extra-cellular sources, are often mediated at the plasma membrane. Membrane associated protein complexes function as the gatekeepers of cell signalling interactions and as such the surface properties of the malignantly transformed cell are demonstrably distinct from their non-cancer counterparts [29]. Re-establishing control of membrane function is an expanding research area for cancer prevention and treatment. Here we highlight how sterols derived from the diet influence membrane structure and function in relation to oncogenic transformation.

\subsubsection{Phytosterol accumulation in cancer prone tissues}

For phytosterols to be considered as potential cancer chemoprevention agents, they must reach and accumulate in tumour prone tissues. Breast tissue contains a variety of phytosterols as assessed by early mass-spectrometry studies [30], which showed that SIT, STIG and CAMP are present in benign breast lesions (ca. $28 \mathrm{ng} \mathrm{sterol/g} \mathrm{tissue;} 18 \mathrm{ng} / \mathrm{g} ; 14 \mathrm{ng} / \mathrm{g}$, respectively), which (NB: assuming a density of tissue equal to that of water) is nearly twice the concentration observed in malignant breast lesions [31]. These observations are in agreement with the hypothesis that phytosterols may be inhibitory to tumour growth and/or may be actively removed during oncogenic progression. Phytosterols also appear to accumulate in other tissues as rats fed phytosterol supplemented diets have significantly higher concentrations in brain [32,33], liver, kidney, erythrocytes and plasma [34], compared to those fed a diet low in phytosterols. This suggests phytosterols can readily enter the circulation and access and accumulate common tumour sites. Updated measurements on larger populations and from different tissue sites and in different cancer subtypes are required to fully understand the pervasiveness (and exclusion during cancer development) of these compounds in mammalian tissues.

\subsubsection{Phytosterol intake and cancer incidence}

Epidemiological data indicate that high phytosterol intake is inversely associated with incidence of several cancer types including that of the endometrium [35], stomach [36] and breast [37]. Similarly, clinical trial data suggest moderate phytosterol intake reduces symptoms in multiple cancer types [38]. In two case control studies of women in New York, dietary phytochemical intake as assessed by food frequency questionnaire (FFQ) demonstrated reduced risk in women with the highest versus lowest intakes of stigmasterol for ovarian cancer (OR 0.42, 95\% CI, 0.20-0.87), and total phytosterols for endometrial cancer (OR 0.6, 95\% CI: 0.3-1.0) [35, 39]. Similarly, a series of case-control studies carried in Uruguay, assessing phytosterol intake retrospectively by questionnaire found significantly reduced risks of lung, gastric and $\mathrm{BCa}[36,40,41]$. In the most recent case-control of Chinese patients, 
the risk of colorectal cancer in those who consumed the highest quartile of phytosterol intake as determined by FFQ was significantly lower than that of the lowest quartile of phytosterol intake (OR $0.50,95 \%$ CI 0.41-0.61) [42].

A recent randomised, double blind control trial using soy milk supplemented with $2 \mathrm{~g}$ phytosterol (sitosterol, stigmasterol and campesterol) or placebo found that after 4 weeks of a daily regime, plasma and urine inflammatory markers such as leukotriene $\mathrm{B} 4$ and $\mathrm{F}_{2}$ isoprostanes, $\mathrm{C}$-Reactive Protein (CRP) and lipid peroxidase activity were significantly reduced [43]. Serum CRP is used clinically as an inflammatory marker, and may be associated with an increased risk of some cancers, in particular, $\mathrm{BCa}$ [44]. Furthermore, in participants given two variants of orange juice beverages supplemented with $2 \mathrm{~g} /$ day phytosterols, significantly attenuated plasma levels of inflammatory markers IL-1b and IL-6 were demonstrated, when compared to placebo [45]. These interleukins are known to be potent inducers of CRP synthesis. Given the known role of inflammatory process, immunomodulation, and reactive species generation in the development of malignancy these data support the hypothesis that phytosterols may be good preventive agents in the primary setting. Furthermore, the indirect relationship between lowering serum CHOL and reduced cancer risk is becoming more apparent [46, 47], highlighted in a recent meta-analysis of statin users demonstrating a protective effect against breast and prostate cancer $[48,49]$. An important benefit of some of these interventional studies is the demonstrable safety and low side-effect profile of short to medium term consumption of up to $2 \mathrm{~g} /$ day of supplemented phytosterols, making them amenable to secondary and tertiary cancer prevention and potentially primary prevention [50-52].

\section{Biophysical interactions of phytosterols with the plasma membrane}

The major structural lipids in mammalian plasma membranes consist of glycerophospholipids (mainly phosphatidylcholine) and sphingolipids (mainly sphingomyelin, with a hydrophobic ceramide backbone) that self-organise to form lipid bilayers. Sterols, i.e. CHOL or phytosterols, are able to regulate the fluidity the biomembrane matrix and can induce phase changes in these membranes [53]. The key membrane parameters to characterise phytosterol interactions with cell surface dynamics fall into three main categories; $i$ ) alterations to lipid rafts - defined as dynamic-ordered transient assemblies enriched in CHOL, sphingolipids and proteins; and caveolae [54] - a variation of membrane raft found in cell surface invaginations, ii) membrane fluidity - the motion of phospholipid molecules within the lipid bilayer, dependent on the structure of phospholipids present, e.g. the composition and length of fatty acid and degree of unsaturation of the acyl chains [55], and iii) membrane curvature - referring to a geometrical measure of curvature [56] (Figure 2). Changes in these parameters can result in alterations to cancer cell behaviour, including proliferation, apoptosis, intra-cellular signalling and metabolism. 


\subsection{Tools for studying phytosterols in membranes}

There are several routes through which these biophysical and cancer cell parameters can be measured experimentally. Biomimetic membranes, whose composition, size and geometry can be tailored, provide highly manipulable models to examine structural alterations to bilayers [57]. Computational modelling can powerfully predict how different sterols may alter these parameters [58], but these are yet to be widely applied to the study of phytosterols. In the field of cancer cell biology, activation of oncogenic signalling pathways can be assessed by measuring changes in the post-translational modifications of pathway proteins, as well as with high throughput transcriptomic and proteomic approaches and other proximity based assays. Sterols in tissue or cells lines can now be directly detected in amounts as low as 25 attograms using spectrometry based methods [59]. Applying LC-MS/MS methods to tissue derived from human volunteers after phytosterol dietary interventions should improve understanding of accumulation of phytosterols in human tissues that are prone to cancer. For example, in an elegant series of lipidomic and cell based assays changes to the lipid content of epithelial cell membranes during polarisation has been reported [60] and a reduction in CHOL content was observed during the epithelial to mesenchymal transition, thus linking plasma membrane components with metastasis, a key hallmark of cancer.

\section{Phytosterols, cholesterol and lipid domains}

Lipid rafts are specialised transient membrane domains thought to be $<200 \mathrm{~nm}$ in size, containing high levels of CHOL and sphingomyelin, as well as other sterols and lipids that facilitate cell signalling complex activation $[61,62]$. CHOL is essential for lipid raft formation with a minimum concentration of 5-8\% CHOL in the fluid phase required before lipid chains are forced into rigid and straight conformations resulting in emergence of the liquid-ordered phase $\left(\mathrm{L}_{\mathrm{o}}\right)$ that is required for raft formation [63]. Crucially, if membrane CHOL coexists with plant sterols, a higher concentration of sterol is required to generate this $\mathrm{L}_{\mathrm{o}}$ phase and there is a reduced propensity for lipid raft formation [64].

Systemic changes in CHOL concentration have also been correlated with altered lipid raft number and type in vivo in prostate tumour xenografts at the cell scale revealing that global $\mathrm{CHOL}$ is an indicator of lipid raft activity. Sensing of extra-cellular signals occurs through proteins embedded within rafts as protein-lipid and protein-protein complexes [62]. Such complexes are more active in cancer cells than in non-cancer cells [65]. CHOL-depletion selectively enhances apoptosis in cancer cells, an effect reversed by CHOL repletion [65]. Active lipid rafts are permissive, perhaps even essential for many oncogenic signalling pathways and many tumour cell lines have been shown to possess an increased number of lipid rafts relative to their non-cancer counter-parts [65]. 


\subsection{Oncogenic signalling via lipid rafts}

The IGF1/PI3K/AKT signalling cascade promotes cancer cell survival, adhesion, migration and proliferation and is dependent on the presence of lipid rafts [66] and one of the most commonly observed cancerous phenotypes. Downstream of PI3K-AKT is mTOR (mammalian Target of Rapamycin), which is a marker of cancer recurrence. In BCa, overexpression of mTOR is associated with a three times greater chance of recurrence [67]. mTOR Complex 1 (mTORC1) is linked to CHOL biosynthesis through its ability to up-regulate the transcription factors SREBF1/2 [68], which are master regulators of fatty acid and CHOL biosynthesis. Feedback between membrane CHOL and mTOR is an emerging area for anti-cancer research [69].

Protein channel opening and closing, and protein movement within rafts is influenced by the lateral pressure generated by sterols. Interactions between membrane forming lipids and proteins in the bilayer are reduced under a higher lateral pressure resulting in lower intra-raft diffusion of proteins and protein pump activity and so the lateral pressure in lipid rafts can influence whether proteins are opening and closing in response to stimuli [70]. This has been demonstrated in the ion pump class of membrane transport proteins [71]. Given that a 'phytosterol membrane sea' produces a weaker lateral pressure than a 'CHOL membrane sea' phytosterols also indirectly regulate proteins embedded in rafts.

Raft formation can be regulated by the molar percentage of CHOL to plant sterols [72] and changes in this ratio that suppress raft-dependent signalling pathways have been observed to impair oncogenic signalling in animal and cell line studies [73]. Exposure to phytosterols is therefore associated with the presence of fewer rafts, presumably due to an increased threshold for lipid ordering (as described in detail below), and fewer rafts leads to diminished pro-survival and anti-apoptotic signalling during oncogenic transformation (Figure 3).

\subsection{Caveolae}

Caveolae are CHOL rich membrane associated invaginations that are packed with signalling proteins and thought to act as lipid raft sinks. Bound to the membrane of these structures is the CAV1 protein that harbours a signaling protein binding sequence allowing recruitment of signalling complexes. Caveolae activity is therefore regulated by phytosterol incorporation into the membrane and several examples of caveolae disruption by phytosterols are observed in the literature. For example, disruption of rafts in Prostate Cancer (PCa) cells with a campesterol and sitosterol mixture promoted disruption of the caveolae, and over-expression of CAV1 inducing p53 tumour suppressor gene activation. The PCa cells entered phytosterol induced and p53 dependent cell cycle arrest and apoptosis [74], revealing one of the molecular mechanisms that could be exploited and tested in cancer prevention and therapy strategies by restoring proper control over the cancer hallmarks of sustained proliferative signalling and evasion of apoptosis [28]. 
Caveolae are particularly important sites for multi-drug resistance (MDR) transport proteins such as the ATP-Binding Cassette (ABC) transporters breast cancer resistance protein (BRCP) and P-glycoprotein (Pgp) [75] and related proteins such as multiple resistance protein (MRP1). These lipid raft embedded MDR proteins export an array of chemotherapy drugs from the cell and MDR is a critical barrier to the successful treatment of many cancers, particularly those where therapy still relies on cytotoxic chemotherapy agents which are readily exported by these ABC pumps. Identifying cancer specific pathways that lead to up-regulation of these proteins is an important area of cancer research as direct inhibitors of the MDR proteins have largely failed in clinical studies owing to their wide-spread requirements in many other tissues such as the blood-brain barrier and intestine; toxicity limits the use of direct MDR inhibitors. BRCP and Pgp-1 were revealed to localise to raft domains in vitro, and increased membranous CHOL was shown to positively modulate their activity [75]. Indeed, coimmunoprecipitation experiments have identified direct physical interactions between CAV1 and MDR proteins such as BCRP. Expression of BCRP is implicated in MDR and poor outcomes for BCa patients undergoing neo-adjuvant chemotherapy [76]. Transcription of another clinically important MDR protein, MRP1, is also regulated at the plasma membrane. The transmembrane Notch-1 protein 'matures' at lipid rich domains [77] resulting in cleavage and release of an intracellular domain which has transcription factor activity $\left(\right.$ Notch $\left.^{\mathrm{ICD}}\right)$. This cleavage of Notch-1 results in cancer specific up-regulation of MRP1 and associated resistance to anthracyclines in BCa cells [78]. Enhanced levels of mature/cleaved Notch-1 generated through aberrant raft formation in cancer cells is also likely to contribute to other oncogenic processes such as enhanced stem cell production [79], proliferation [80] and angiogenesis [81].

\subsection{The sphingomyelin cycle}

Phytosterols appear to play a role in altering the composition of key raft components such as CHOL and sphingomyelin. In HT-29 colon cancer cells that were growth inhibited by supplementation with $16 \mu \mathrm{M}$ SIT, a $26 \%$ reduction of membrane $\mathrm{CHOL}$, as well as a $50 \%$ reduction in membrane sphingomyelin was observed, without changes in total membrane phospholipid concentration [82]. This study led the way for a host of experimentation in understanding phytosterol related membrane changes, focused in particular on the synthesis of ceramide via the sphingomyelin cycle. Ceramide is a key constituent of sphingolipid metabolism and the head component of the core lipid raft molecule, sphingomyelin. It has tumour-suppressing capacity, inducing and mediating apoptotic and antiproliferative responses in numerous cancer cell types both through its presence in the membrane and inside the cell [83]. In LnCAP prostate cancer cells in vitro supplemented with SIT display decreases in membrane $\mathrm{CHOL}$ and sphingomyelin increased ceramide levels and up-regulation of protein phosphatase $2 \mathrm{~A}$, a target of ceramide's anti-survival influence on key apoptotic regulator Bc12 [84, 85]. 
SIT supplementation in combination with Tamoxifen resulted in growth inhibition of two BCa cell lines that was greater than either regimen individually [86]. The growth inhibition was accompanied with a synergistic increase in ceramide levels as had previously reported in prostate and colon cancer cell lines. Awad and colleagues were able to show SIT induced serine palmitoyltransferase activity, a rate-limiting enzyme in sphingolipid metabolism and ceramide synthesis, thus demonstrating the anti-cancer properties of SIT at least partially required the ceramide metabolic pathway. An increase of endogenous ceramide at the expense of CHOL in normal Schwann cell membranes in vitro was generated by exogenous supplementation with bacterial sphingomyelinase [87] and SIT supplementation led to increase of ceramide in model membranes at the expense of CHOL and led to lipid segregation and membrane organisational changes [88]. Collectively, these results indicate that SIT can impair oncogenic signal transduction by depleting raft $\mathrm{CHOL}$ via changes to ceramide-mediated membrane signalling (Figure 3). Importantly, these results also indicate that phytosterol induced ceramide synthesis may be protect against oncogenic transformation.

\section{Membrane fluidity and molecular packing}

A number of studies using biophysical approaches such as electron paramagnetic resonance (EPR), fluorescence spectroscopy and nuclear magnetic resonance spectroscopy (NMR) have been used to elucidate the behaviour of the plasma membrane in the presence of CHOL [89-92]. Synthetic membranes are commonly comprised of a mixture of lipids (combinations of DMPC, DPPC or POPC) further mixed with $\mathrm{CHOL}$ or other sterols.

The interactions of phytosterols with model membranes have been extensively studied over the years with their effects commonly compared to that of CHOL [64, 93-96]. Small angle X-ray scattering (SAXS) can be applied to determine changes in bilayer thickness, effective packing area per lipid, and any undulation that occurs when lipid bilayers are formed in the presence of different sterols. SAXS analysis of the binary mixtures of lipid/sterol systems indicate a uniform phase when sterol concentrations were below 5\% with no evidence for the existence of lipid rafts, as in agreement with several other studies $[97,98]$.

CHOL introduces a high degree of conformational order on the acyl tails of lipids in the fluid phases [99, 100], known as the 'ordering effect' (liquid ordered $\mathrm{L}_{\mathrm{o}}$ phase formation). The $\mathrm{L}_{\mathrm{o}}$ phase reflects a tightly packed bilayer with increased membrane rigidity and thickness (Figure 2). In contrast, in the gel phase $\left(\mathrm{L}_{\beta}\right)$, CHOL exhibits opposite effects on membrane, i.e. renders the $\mathrm{L}_{\beta}$ phase membrane less ordered [99, 100]. Atomic Force Microscopy (AFM) has been used to evaluate lipid organisation and ordering, leading to experimental observation of three phases including this disordered gel phase, $\mathrm{L}_{\mathrm{d}}$ [101]. Importantly for cancer therapy research, the presence of $L_{d}$ may be essential for the delivery of some cancer therapeutics such as paclitaxel [102]. 
SAXS studies with CHOL, SIT and STIG revealed that CHOL, independent of the lipid composition of the membrane, most significantly altered the membrane structural parameters, such as bilayer thickness and fluctuations, followed by SIT and STIG (Figure 2). Less efficient membrane modulations with SIT and STIG have been attributed to their structural variations, i.e. both sterols contain an additional ethyl group at $\mathrm{C} 24$ in the side chain when compared to CHOL [103, 104]. These structural features were suggested to render these sterols less flexible for their interaction with phospholipids [64]. STIG, ERGO and BRASS also have a double bond in the side chain $\left(\mathrm{C}_{22-23}\right)$ making them more flexible than other plant sterols with saturated side chains such as SIT and CAMP (Figure 1). The rotational rigidity imposed by saturation of this double bond in CHOL, CAMP and SIT preferentially leads to the $\mathrm{L}_{\mathrm{o}}$ phase formation whereas STIG decreases order [105]. It is plausible to expect that SAXS and/or computation modelling experiments may reveal sterols with this double bond would have a thinner bilayer, and looser lipid packing. So whilst CAMP and SIT lead to a relatively small change in parameters associated with disrupting lipid raft formation and membrane fluidity, their unsaturated counterparts (STIG, BRASS) are more effective at increasing membrane fluidity [106].

The membrane ordering imposed by CAMP was also shown to be comparable to that of CHOL when assessed using fluorescent probes and confocal microscopy [96]. These experiments indicated that if CAMP were to fully replace CHOL in the membrane, rigidity may be altered relatively little. SIT behaved similarly to STIG and induced far less ordering than CAMP and CHOL (Figure 2) suggesting biophysical properties other than the bond saturation were responsible in these experiments. Such a strong membrane ordering effect is in agreement with other studies where CAMP caused closer packing in liposomal bilayers of lecithin and sphingomyelin (48mol\%:13mol\%) giving rise to an increased membrane rigidity [107]. Similarly, CAMP demonstrated a high ability to stabilize both DPPC and PSM bilayers compared to both STIG and $\beta$-SIT [94]. These studies further support the notion that the effect of CAMP on packing tightness is attributed to its reduced rotational freedom in the $\mathrm{C}_{17^{-}}$ hydrocarbon chain. STIG has been less capable of membrane ordering than other sterols studied, and therefore predictably lacks the ability to promote the formation of $L_{o}$ phase domains $[108,109]$. The saturation of the plant sterol side chain is a key parameter of the sterol's potential to alter the biophysical properties of lipid bilayers $[72,106]$ and is therefore an interesting parameter to perturb in computational models of phytosterol based plasma membranes.

\subsection{The balance between fluidity and rigidity in cancer cell membranes}

The balance between membrane fluidity and rigidity is an important consideration in cancer therapy and prevention. A highly rigid membrane provides an efficient barrier to passive drug diffusion across membranes of water soluble drugs and with an increase on the lateral membrane pressure also suppresses the functionality of proteins within lipid rafts. Contrary to this is the observation that migratory metastatic cells have a more fluid membrane, allowing them to better navigate cell-cell 
junctions and gaps in basement membranes. So whilst high rigidity is perhaps an indicator of poor drug responsiveness, low rigidity is more likely to be associated with cancer spread. One of the earliest systematic studies of membrane fluidity regulation of soybean PC bilayers with CHOL and plant sterols monitored membrane fluidity alterations with a range of phytosterols; SIT, CAMP, STIG, cycloartenol, 24-methylpollinastanol and 14alpha,24-dimethylcholest-8-en-3beta-ol through the steady-state fluorescence anisotropy measurements and the 1,6-diphenyl-1,3,5-hexatriene (DPH) [108]. Cycloartenol exhibited a strong ordering ability similar to that of CHOL [108] suggesting that its incorporation into membranes may prevent cancer cell mobility. In addition, hydrocarbon chain ordering, (a key step for the formation of the liquid ordered phase $\left(\mathrm{L}_{\mathrm{o}}\right)$, which in turn is a prerequisite for raft formation) of soybean PC bilayers was the strongest with CAMP and SIT, and the least with STIG, 24-methylpollinastanol and 14alpha,24-dimethylcholest-8-en-3beta-ol.

In vitro experiments have also been performed to evaluate membrane rigidity. Erythrocytes from rats fed with canola oil, abundant in phytosterols, possessed significantly higher ratios of phytosterol/CHOL and a significantly lower membrane deformability index to those fed with soybean oil less abundant in phytosterols [34]. This appears at first to be in contrast to the observations described above where phytosterol incorporation into the membrane results in a less rigid membrane than if CHOL was introduced. However, some sterols with saturated side-chains such as those found in canola, are likely to induce membrane stiffness. This may have important implications to the cancer cell. Primary breast and cervical cancer cells have been demonstrated to have lower membrane rigidity than their normal counterparts, and ovarian cancer cell lines with the highest potential to undergo metastatic changes have up to five times less stiffness $(\mathrm{Pa})$ than those with the lowest $[110,111]$. In fact, treatment of MDAMB-231 BCa cells with Soraphen A to induce membrane rigidity was found to weaken the ability of these cells to migrate and invade [112]. If phytosterols without saturated side-chains were to have the same effect and reduce fluidity in cancer cells, this may explain why phytosterol treatment reduces the metastatic and invasive properties of MDA-MB-231 cells in vitro, and in SCID mice [113, 114].

There may be further evidence for a relationship between plasma membrane fluidity changes and the protective potentials of plant sterols against BCa. Two widely used cancer drugs; doxorubicin (DOX) and paclitaxel (PTF) in the advanced and metastatic BCa setting are thought to integrate with DNA and disrupt the cytoskeleton, but they also alter the membrane fluidity. Both drugs enhanced the fluidity at lower concentration ranges $(0.5-5 \mu \mathrm{M})$ but conferred a rigidifying effect at high concentrations (5-20 $\mu \mathrm{M})$. Changes in membrane fluidity induced by DOX and PTF are associated with drug penetration into the cell membrane [115]. This could have important implications for cancer therapy where passive diffusion of cytotoxic agents into cancer cells can be hampered by rigid and tightly packed plasma membranes and the incorporation of specific plant sterols, which one would predict to have saturated side-chains, could improve drug uptake. 
Improved understanding of how plant sterols alter bilayer fluidity is key to linking therapeutic potential with a solid robust understanding of their molecular mechanisms. It is plausible that if phytosterols are specifically able to increased membrane fluidity they may be therapeutically applicable during systemic chemotherapy treatment, where they could antagonise the rigid membrane barrier that prevents passive drug diffusion. However, the same process may exacerbate cell motility by increasing fluidity and the potential for cells to traverse basement membranes and junctions so different sterols may be applicable at different stages of treatment.

\section{Membrane curvature and asymmetry}

Due to the non-random distribution of phospholipids between the outer and inner leaflets of the membranes, the cell membrane is considered as an asymmetric structure and this asymmetry contributes to membrane curvature. So far however, very few studies focused on the behaviour of CHOL or phytosterols in asymmetric membranes owing to the difficulty of generating asymmetrical biosynthetic membranes and this is a critical area for future development. Several studies have however assessed curvature. Kollmitzer and colleagues probed induction of spontaneous curvature $\left(J_{0}\right)$ with CHOL and a range of biologically relevant phospholipids within inverted hexagonal host-phase using SAXS [95]. The mixtures of POPC/eggSM/CHOL and DOPC/DSPC/CHOL resulted in a more negative curvature in the $\mathrm{L}_{\mathrm{o}}$ phase compared to the coexisting liquid disordered $\mathrm{L}_{d}$ phase. In contrast, DOPC/DPPC/CHOL exhibited a more positive curvature in the liquid ordered phase. A positively curved membrane would favour partitioning of barrel-shaped proteins into the liquid ordered phase. HSP70 is one such barrelshaped protein and is tethered to the membrane in CHOL rich raft-like regions. Expression of HSP70 is elevated in several tumour types and is an emerging therapeutic target for antibody therapy [116].

Alterations in membrane curvature can possibly lead to consequences of membrane restructuring for protein function, e.g. ion channel activity. Structural analysis of POPC/SM/CHOL/Ceramide membranes with $10 \mathrm{~mol} \% \mathrm{CHOL}$ showed a tighter lipid packing with an increase in the membrane thickness in the lamellar fluid phase in comparison to CHOL-free membranes [117, 118]. Comparison of the ion channel activity through theoretical calculations implied that channel opening occurs in domains of CHOL-free membranes, whereas the presence of CHOL helped maintain membrane stability and function. These observations suggest that the absence of CHOL induces strong alterations in the conformational equilibrium of membrane proteins, which could impair transport out of the cell and membrane associated signalling and function, and may interfere with the controlled disposal of cells during apoptosis. Importantly, such alterations in the conformational equilibrium of membrane proteins were much smaller in the presence of CHOL [119]. 


\section{Emerging areas for phytosterol cancer research}

\subsection{Computational modelling}

The phytosterols are a sterol class with over 200 known species and represent a natural library of sterol structural parameters with variation in many biophysical properties. A systematic assessment of how side-chain flexibility, bond saturation, length, and hydroxylation alter membrane ordering would lead to predictions as to exactly which properties are most important in modulating intra-cellular signalling and in membrane structure and function.

The field of atomic computational modelling allows the experimental manipulation of such parameters [58]. The current limitation is that existing sterol databases [120] contain ergosterol but few other phytosterol-like compounds limiting the application of computational models to plant compounds. For these computational experiments to take place, systematic parameterisation of phytosterols needs to be performed and these annotated phytosterols then added to public repositories such as cgmartini.nl [121]. The dissemination of these parameterised descriptions of phytosterols would allow more computational laboratories to begin evaluating phytosterols in their membrane models. With sufficient progress in simulating accurately the complex variability of the membrane, this could provide an alternative avenue to further explore the impact of phytosterols in advance of in vitro or in vivo study [58]. Specific examples of how computational modelling could enhance phytosterol-membrane understanding are provided by atomistic modelling [122] of how oxysterols regulate membrane CHOL availability and curvature prediction. The oxysterol 25-OHC appears to increase CHOL availability which indicates 25OHC pushes CHOL to the membrane surface exposing it to cellular receptors or enzymes. If such modelling experiments could be applied to phytosterols they may reveal whether phytosterols alter oxysterol production, by controlling $\mathrm{CHOL}$ exposure to membrane tethered enzymes such as the cytochrome P450 family. Oxysterols have themselves been implicated in driving chemotherapy resistance [123] and metastasis [47]. Computational prediction of membrane curvature induced by phytosterols could be useful to infer changes in signalling mediated by partitioning of barrel shaped proteins such as the HSP family members. Whilst a systematic 'wet-lab' evaluation of phytosterols, either through biosynthetic membranes or cancer cell biology assays, would be restricted by the costs associated with obtaining purified sterols, computational simulations are restricted by the time taken to fully ascribe parameters to individual phytosterols and are limited to laboratories with advanced computing capability. A balance between approaches, and collaboration between fields, will allow iterative refinement of experiments that would allow the most efficient use of resources to better investigate promising anti-cancer agents.

\subsection{Drug resistance in clinic}

At the clinical level, membranous CHOL depletion by phytosterols may prove an exciting new avenue in combatting drug resistance in patients. Phytosterol mediated CHOL depletion would prevent 
'maturation' of the membrane bound oncogenes Notch-1 thus stopping translocation to the nucleus where they would otherwise up-regulate MDR proteins and promote stem cell features. Reduced lipid raft formation would also impair $\mathrm{ABC}$ transport protein function again leading to enhanced retention of anti-cancer cytotoxic agents within tumour cells. Importantly this would depend on reaching critical concentrations of phytosterols in the plasma membrane of tumour cells, this threshold remains to be defined in vivo.

\subsection{Improved accuracy in sterol differentiation, quantification and detection}

Resolving sterols that have very similar structures, molecular weights and polarity on chromatograms is a challenge that is being addressed by analytical chemists. The emergence of highly sensitive LCMS/MS methodologies now allows attogram level detection of oxysterols, cholesterol metabolites that are biophysically similar to phytosterols. The adaptation of these methods to quantifying phytosterols will allow researchers to make highly accurate measurements in human tissue and cell lines to determine at what concentration lipid raft formation is prevented and/or when oncogenic signalling is impaired in cell based assays. Of particular interest would be assessment of local phytosterol concentrations that appear to limit raft formation. Future studies could assess phytosterol content of membranes of tissues obtained from volunteers undergoing phytosterol dietary interventions and the penetration of phytosterols into tumour prone tissues requires clarification. Several bio-banks are now collecting tissue samples from volunteers [124] and an adaptation to protocols to allow a pre-/post-intervention comparison would provide a useful resource for researchers interested in assessing the accumulation and biological impact of dietary agents in tissues and their integration into the cell.

\section{Summary}

Phytosterols are proposed as potential cancer prevention agents because of the range of molecular routes through which the antagonise oncogenic pathways, and also because of the ease through which they can be administered to large populations. Although the molecular and clinical evidence to conclusively define them as anti-cancer agents remains limited, these features warrant further investigation at the clinical trial level in at risk populations.

Phytosterols integrate into normal and cancer cell membranes and replace CHOL producing substantial changes in the 3D structure and biophysical properties. Phytosterols also reduce systemic CHOL, thus acting both directly (through integration into the plasma membrane) and indirectly (through restricting the supply of CHOL) to alter membrane biophysical properties. Generally, replacement of CHOL with phytosterols in the membrane leads to altered lipid raft formation, reduced stiffness of the membrane and changes to the lateral pressure profile. These features are consistent with reduced oncogenic signalling and suppression of the classical cancer hallmarks. Nevertheless, there remains a scarcity of trial evidence examining phytosterol supplementation and cancer prevention and a scarcity of human 
studies where plasma membranes have been evaluated after phytosterol administration. In the cancer prevention setting, the costs of the necessary large scale studies to prove a compound has cancer preventive properties is prohibitive. Observational and questionnaire based assessment of phytosterol intake and cancer incidence and outcomes however do emphasise the need for sufficiently focused, powered and well-designed RCTs to evaluate phytosterols as cancer prevention agents, initially in the tertiary setting. Biomimetic membranes and in vitro analysis of cell systems have been strongly researched in the past and will continue to be useful in evaluating different sterols. But now, in combination with the emerging areas of ex vivo culture of human tissue biopsies following dietary interventions, and computational modelling to explore how phytosterols with their array of structural properties alter membrane properties, these older and established methodologies offer new opportunities to evaluate phytosterols as agents for the clinical management of cancer.

\section{ACKNOWLEDGEMENTS}

The authors wish to thank Prof Michael Rappolt and Dr Antreas Kalli for their critical feedback on the manuscript. We also wish to thank the ENOR organising committee for the opportunity to publish this review.

\section{FUNDING SOURCES}

Dr Thorne is supported by the School of Food Science and Nutrition at the University of Leeds and through generous research grants from Breast Cancer Research Action Group [Reference Number: 3T57/9R17-02] which is part of the Leeds Charitable Foundation, and Breast Cancer UK [Reference Number: RF_MA_Oxy/PO 1605-382].

\section{FIGURE LEGENDS}

Figure 1. Chemical structures of sterols. The sterols usually possess C27-C29 skeletons, with differences in alkylation at $\mathrm{C} 24$ and double bonds in the nucleus $(\Delta 5, \Delta 8, \Delta 8(14))$ and side chain $(\Delta 22, \Delta 24, \Delta 24(28)$. The most common position of the double bond is C5 followed by C22 and C7. Compared to Cholesterol, Sitosterol and Stigmasterol contain an additional ethyl group at C24 in the side chain. Stigmasterol, Ergosterol and Brassicasterol have a double bond in the side chain (C22-C23) making them more flexible than other plant sterols with saturated side chains.

\section{Figure 2: Variations to membrane properties caused by different sterols.}

Figure 3: The avenues for phytosterol mediated membrane changes and their proposed effects on malignant cell viability. Phytosterols act in a dual fashion to deplete membrane cholesterol, and therefore impair lipid raft formation. Through overexpression of CAV1, a tumour suppressor gene, phytosterols disrupt caveolae and up-regulate $\mathrm{BCl}-\mathrm{Xs}$ and p53 - 
promoting apoptosis in malignant cells. Ceramide synthesis also depletes membrane cholesterol, and can similarly up-regulate p53.

References

[1] J. Schuz, C. Espina, P. Villain, R. Herrero, M.E. Leon, S. Minozzi, I. Romieu, N. Segnan, J. Wardle, M. Wiseman, F. Belardelli, D. Bettcher, F. Cavalli, G. Galea, G. Lenoir, J.M. MartinMoreno, F.A. Nicula, J.H. Olsen, J. Patnick, M. Primic-Zakelj, P. Puska, F.E. van Leeuwen, O. Wiestler, W. Zatonski, E. Working Groups of Scientific, European Code against Cancer 4th Edition: 12 ways to reduce your cancer risk, Cancer Epidemiol, 39 Suppl 1 (2015) S1-10. [2] S.G. Nayfield, J.E. Karp, L.G. Ford, F.A. Dorr, B.S. Kramer, Potential role of tamoxifen in prevention of breast cancer, Journal of the National Cancer Institute, 83 (1991) 1450-1459. [3] V.C. Jordan, J.I. MacGregor, D.A. Tonetti, Tamoxifen: from breast cancer therapy to the design of a postmenopausal prevention maintenance therapy, Clin Oncol (R Coll Radiol), 9 (1997) 390-394.

[4] V.C. Jordan, Tamoxifen for breast cancer prevention, Proc Soc Exp Biol Med, 208 (1995) 144-149.

[5] P. Chen, C.C. Yang, Y.J. Chen, P.H. Wang, Tamoxifen-induced endometrial cancer, Eur J Gynaecol Oncol, 24 (2003) 135-137.

[6] C. Bosetti, V. Rosato, S. Gallus, J. Cuzick, C. La Vecchia, Aspirin and cancer risk: a quantitative review to 2011, Annals of oncology : official journal of the European Society for Medical Oncology / ESMO, 23 (2012) 1403-1415. 
[7] M.A. Thorat, J. Cuzick, Role of aspirin in cancer prevention, Curr Oncol Rep, 15 (2013) 533-540.

[8] S.G. Smith, R. Foy, J. McGowan, L.C. Kobayashi, J. Burn, K. Brown, L. Side, J. Cuzick, General practitioner attitudes towards prescribing aspirin to carriers of Lynch Syndrome: findings from a national survey, Fam Cancer, 16 (2017) 509-516.

[9] M.A. Thorat, J. Cuzick, Prophylactic use of aspirin: systematic review of harms and approaches to mitigation in the general population, Eur J Epidemiol, 30 (2015) 5-18.

[10] V. Escurriol, M. Mari-Dell'Olmo, I. Rohlfs, C. Borrell, M.D. Chirlaque, G. Buckland, L. Rodriguez, M.J. Sanchez, P. Amiano, N. Egues, E. Ros, Plant sterol intake and education level in the Spanish EPIC cohort, Nutrition, 25 (2009) 769-773.

[11] S. Klingberg, H. Andersson, A. Mulligan, A. Bhaniani, A. Welch, S. Bingham, K.T. Khaw, S. Andersson, L. Ellegard, Food sources of plant sterols in the EPIC Norfolk population, European journal of clinical nutrition, 62 (2008) 695-703.

[12] S. Pinedo, M.N. Vissers, K. von Bergmann, K. Elharchaoui, D. Lutjohann, R. Luben, N.J. Wareham, J.J. Kastelein, K.T. Khaw, S.M. Boekholdt, Plasma levels of plant sterols and the risk of coronary artery disease: the prospective EPIC-Norfolk Population Study, Journal of lipid research, 48 (2007) 139-144.

[13] M.M. Best, C.H. Duncan, E.J. Van Loon, J.D. Wathen, Lowering of serum cholesterol by the administration of a plant sterol, Circulation, 10 (1954) 201-206.

[14] R. Brauner, C. Johannes, F. Ploessl, F. Bracher, R.L. Lorenz, Phytosterols reduce cholesterol absorption by inhibition of 27-hydroxycholesterol generation, liver $\mathrm{X}$ receptor alpha activation, and expression of the basolateral sterol exporter ATP-binding cassette A1 in Caco2 enterocytes, J Nutr, 142 (2012) 981-989.

[15] B.A. Carter, O.A. Taylor, D.R. Prendergast, T.L. Zimmerman, R. Von Furstenberg, D.D. Moore, S.J. Karpen, Stigmasterol, a soy lipid-derived phytosterol, is an antagonist of the bile acid nuclear receptor FXR, Pediatr Res, 62 (2007) 301-306.

[16] E.R. Nelson, C.D. DuSell, X. Wang, M.K. Howe, G. Evans, R.D. Michalek, M. Umetani, J.C. Rathmell, S. Khosla, D. Gesty-Palmer, D.P. McDonnell, The oxysterol, 27hydroxycholesterol, links cholesterol metabolism to bone homeostasis through its actions on the estrogen and liver $X$ receptors, Endocrinology, 152 (2011) 4691-4705.

[17] N. Pencheva, C.G. Buss, J. Posada, T. Merghoub, S.F. Tavazoie, Broad-Spectrum Therapeutic Suppression of Metastatic Melanoma through Nuclear Hormone Receptor Activation, Cell, 156 (2014) 986-1001.

[18] H. de Boussac, A.J. Pommier, J. Dufour, A. Trousson, F. Caira, D.H. Volle, S. Baron, J.M. Lobaccaro, LXR, prostate cancer and cholesterol: the Good, the Bad and the Ugly, Am J Cancer Res, 3 (2013) 58-69. 
[19] I. Demonty, R.T. Ras, H.C. van der Knaap, G.S. Duchateau, L. Meijer, P.L. Zock, J.M. Geleijnse, E.A. Trautwein, Continuous dose-response relationship of the LDL-cholesterollowering effect of phytosterol intake, J Nutr, 139 (2009) 271-284.

[20] E. National Cholesterol Education Program Expert Panel on Detection, A. Treatment of High Blood Cholesterol in, Third Report of the National Cholesterol Education Program (NCEP) Expert Panel on Detection, Evaluation, and Treatment of High Blood Cholesterol in Adults (Adult Treatment Panel III) final report, Circulation, 106 (2002) 3143-3421.

[21] S.W. Andersson, J. Skinner, L. Ellegard, A.A. Welch, S. Bingham, A. Mulligan, H. Andersson, K.T. Khaw, Intake of dietary plant sterols is inversely related to serum cholesterol concentration in men and women in the EPIC Norfolk population: a cross-sectional study, European journal of clinical nutrition, 58 (2004) 1378-1385.

[22] V. Escurriol, M. Cofan, C. Moreno-Iribas, N. Larranaga, C. Martinez, C. Navarro, L. Rodriguez, C.A. Gonzalez, D. Corella, E. Ros, Phytosterol plasma concentrations and coronary heart disease in the prospective Spanish EPIC cohort, Journal of lipid research, 51 (2010) 618-624.

[23] R.T. Ras, Y.T. van der Schouw, E.A. Trautwein, I. Sioen, G.W. Dalmeijer, P.L. Zock, J.W. Beulens, Intake of phytosterols from natural sources and risk of cardiovascular disease in the European Prospective Investigation into Cancer and Nutrition-the Netherlands (EPIC-NL) population, Eur J Prev Cardiol, 22 (2015) 1067-1075.

[24] M.S. Bosner, L.G. Lange, W.F. Stenson, R.E. Ostlund, Jr., Percent cholesterol absorption in normal women and men quantified with dual stable isotopic tracers and negative ion mass spectrometry, Journal of lipid research, 40 (1999) 302-308.

[25] R.G. Gould, R.J. Jones, G.V. LeRoy, R.W. Wissler, C.B. Taylor, Absorbability of betasitosterol in humans, Metabolism: clinical and experimental, 18 (1969) 652-662.

[26] M.J. Mellies, T.T. Ishikawa, P.S. Gartside, K. Burton, J. MacGee, K. Allen, P.M. Steiner, D. Brady, C.J. Glueck, Effects of varying maternal dietary fatty acids in lactating women and their infants, The American journal of clinical nutrition, 32 (1979) 299-303.

[27] A.R. Stiles, J. Kozlitina, B.M. Thompson, J.G. McDonald, K.S. King, D.W. Russell, Genetic, anatomic, and clinical determinants of human serum sterol and vitamin D levels, Proc Natl Acad Sci U S A, 111 (2014) E4006-4014.

[28] D. Hanahan, R.A. Weinberg, Hallmarks of cancer: the next generation, Cell, 144 (2011) 646-674.

[29] K. Kojima, Molecular aspects of the plasma membrane in tumor cells, Nagoya J Med Sci, 56 (1993) 1-18.

[30] G.S. Gordan, M.E. Fitzpatrick, W.P. Lubich, Identification of osteolytic sterols in human breast cancer, Trans Assoc Am Physicians, 80 (1967) 183-189. 
[31] M.J. Mellies, T.T. Ishikawa, C.J. Glueck, J.D. Crissman, Phytosterols and cholesterol in malignant and benign breast tumors, Cancer research, 37 (1977) 3034-3036.

[32] P.J. Jansen, D. Lutjohann, K. Abildayeva, T. Vanmierlo, T. Plosch, J. Plat, K. von Bergmann, A.K. Groen, F.C. Ramaekers, F. Kuipers, M. Mulder, Dietary plant sterols accumulate in the brain, Biochim Biophys Acta, 1761 (2006) 445-453.

[33] T. Vanmierlo, O. Weingartner, S. van der Pol, C. Husche, A. Kerksiek, S. Friedrichs, E. Sijbrands, H. Steinbusch, M. Grimm, T. Hartmann, U. Laufs, M. Bohm, H.E. de Vries, M. Mulder, D. Lutjohann, Dietary intake of plant sterols stably increases plant sterol levels in the murine brain, J Lipid Res, 53 (2012) 726-735.

[34] W.M. Ratnayake, M.R. L'Abbe, R. Mueller, S. Hayward, L. Plouffe, R. Hollywood, K. Trick, Vegetable oils high in phytosterols make erythrocytes less deformable and shorten the life span of stroke-prone spontaneously hypertensive rats, J Nutr, 130 (2000) 1166-1178.

[35] S.E. McCann, J.L. Freudenheim, J.R. Marshall, J.R. Brasure, M.K. Swanson, S. Graham, Diet in the epidemiology of endometrial cancer in western New York (United States), Cancer causes \& control : CCC, 11 (2000) 965-974.

[36] E. De Stefani, P. Boffetta, A.L. Ronco, P. Brennan, H. Deneo-Pellegrini, J.C. Carzoglio, M. Mendilaharsu, Plant sterols and risk of stomach cancer: a case-control study in Uruguay, Nutrition and cancer, 37 (2000) 140-144.

[37] L. Torres-Sanchez, M. Galvan-Portillo, M.S. Wolff, L. Lopez-Carrillo, Dietary consumption of phytochemicals and breast cancer risk in Mexican women, Public Health Nutr, 12 (2009) 825-831.

[38] N. Shahzad, W. Khan, S. Md, A. Ali, S.S. Saluja, S. Sharma, F.A. Al-Allaf, Z. Abduljaleel, I.A.A. Ibrahim, A.F. Abdel-Wahab, M.A. Afify, S.S. Al-Ghamdi, Phytosterols as a natural anticancer agent: Current status and future perspective, Biomed Pharmacother, 88 (2017) 786-794.

[39] S.E. McCann, J.L. Freudenheim, J.R. Marshall, S. Graham, Risk of human ovarian cancer is related to dietary intake of selected nutrients, phytochemicals and food groups, J Nutr, 133 (2003) 1937-1942.

[40] M. Mendilaharsu, E. De Stefani, H. Deneo-Pellegrini, J. Carzoglio, A. Ronco, Phytosterols and risk of lung cancer: a case-control study in Uruguay, Lung Cancer, 21 (1998) 37-45.

[41] A. Ronco, E. De Stefani, P. Boffetta, H. Deneo-Pellegrini, M. Mendilaharsu, F. Leborgne, Vegetables, fruits, and related nutrients and risk of breast cancer: a case-control study in Uruguay, Nutr Cancer, 35 (1999) 111-119.

[42] J. Huang, M. Xu, Y.J. Fang, M.S. Lu, Z.Z. Pan, W.Q. Huang, Y.M. Chen, C.X. Zhang, Association between phytosterol intake and colorectal cancer risk: a case-control study, $\mathrm{Br} \mathrm{J}$ Nutr, 117 (2017) 839-850. 
[43] X.L. Ho, J.J. Liu, W.M. Loke, Plant sterol-enriched soy milk consumption modulates 5lipoxygenase, 12-lipoxygenase, and myeloperoxidase activities in healthy adults - a randomized-controlled trial, Free Radic Res, 50 (2016) 1396-1407.

[44] L. Guo, S. Liu, S. Zhang, Q. Chen, M. Zhang, P. Quan, J. Lu, X. Sun, C-reactive protein and risk of breast cancer: A systematic review and meta-analysis, Sci Rep, 5 (2015) 10508.

[45] S. Devaraj, I. Jialal, J. Rockwood, D. Zak, Effect of orange juice and beverage with phytosterols on cytokines and PAI-1 activity, Clin Nutr, 30 (2011) 668-671.

[46] E.R. Nelson, C.Y. Chang, D.P. McDonnell, Cholesterol and breast cancer pathophysiology, Trends Endocrinol Metab, 25 (2014) 649-655.

[47] E.R. Nelson, S.E. Wardell, J.S. Jasper, S. Park, S. Suchindran, M.K. Howe, N.J. Carver, R.V. Pillai, P.M. Sullivan, V. Sondhi, M. Umetani, J. Geradts, D.P. McDonnell, 27Hydroxycholesterol links hypercholesterolemia and breast cancer pathophysiology, Science, 342 (2013) 1094-1098.

[48] B. Liu, Z. Yi, X. Guan, Y.X. Zeng, F. Ma, The relationship between statins and breast cancer prognosis varies by statin type and exposure time: a meta-analysis, Breast Cancer Res Treat, 164 (2017) 1-11.

[49] X. Liu, J. Li, S.E. Schild, M.H. Schild, W. Wong, S. Vora, M.G. Herman, M. Fatyga, Statins and Metformin Use Is Associated with Lower PSA Levels in Prostate Cancer Patients Presenting for Radiation Therapy, J Cancer Ther, 8 (2017) 73-85.

[50] K.C. Maki, A.L. Lawless, M.S. Reeves, M.R. Dicklin, B.H. Jenks, E. Shneyvas, J.R. Brooks, Lipid-altering effects of a dietary supplement tablet containing free plant sterols and stanols in men and women with primary hypercholesterolaemia: a randomized, placebo-controlled crossover trial, Int J Food Sci Nutr, 63 (2012) 476-482.

[51] H. Gylling, J. Plat, S. Turley, H.N. Ginsberg, L. Ellegard, W. Jessup, P.J. Jones, D. Lutjohann, W. Maerz, L. Masana, G. Silbernagel, B. Staels, J. Boren, A.L. Catapano, G. De Backer, J. Deanfield, O.S. Descamps, P.T. Kovanen, G. Riccardi, L. Tokgozoglu, M.J. Chapman, P. European Atherosclerosis Society Consensus Panel on, Plant sterols and plant stanols in the management of dyslipidaemia and prevention of cardiovascular disease, Atherosclerosis, 232 (2014) 346-360.

[52] V.Z. Rocha, R.T. Ras, A.C. Gagliardi, L.C. Mangili, E.A. Trautwein, R.D. Santos, Effects of phytosterols on markers of inflammation: A systematic review and meta-analysis, Atherosclerosis, 248 (2016) 76-83.

[53] G. van Meer, D.R. Voelker, G.W. Feigenson, Membrane lipids: where they are and how they behave, Nat Rev Mol Cell Bio, 9 (2008) 112-124.

[54] H.H. Patel, P.A. Insel, Lipid Rafts and Caveolae and Their Role in Compartmentation of Redox Signaling, Antioxidants \& Redox Signaling, 11 (2009) 1357-1372. 
[55] P.V. Escribá, J.M. González-Ros, F.M. Goñi, P.K.J. Kinnunen, L. Vigh, L. SánchezMagraner, A.M. Fernández, X. Busquets, I. Horváth, G. Barceló-Coblijn, Membranes: a meeting point for lipids, proteins and therapies, Journal of Cellular and Molecular Medicine, 12 (2008) 829-875.

[56] H.T. McMahon, E. Boucrot, Membrane curvature at a glance, Journal of Cell Science, 128 (2015) 1065-1070.

[57] M. Eeman, M. Deleu, From biological membranes to biomimetic model membranes, Biotechnol Agron Soc, 14 (2010) 719-736.

[58] W.F. Bennett, D.P. Tieleman, Computer simulations of lipid membrane domains, Biochimica et biophysica acta, 1828 (2013) 1765-1776.

[59] T. Vehus, H. Roberg-Larsen, J. Waaler, S. Aslaksen, S. Krauss, S.R. Wilson, E. Lundanes, Versatile, sensitive liquid chromatography mass spectrometry - Implementation of 10 mum OT columns suitable for small molecules, peptides and proteins, Scientific reports, 6 (2016) 37507. [60] J.L. Sampaio, M.J. Gerl, C. Klose, C.S. Ejsing, H. Beug, K. Simons, A. Shevchenko, Membrane lipidome of an epithelial cell line, Proc Natl Acad Sci U S A, 108 (2011) 1903-1907. [61] L.J. Pike, Lipid rafts: bringing order to chaos, J Lipid Res, 44 (2003) 655-667.

[62] E. Sezgin, I. Levental, S. Mayor, C. Eggeling, The mystery of membrane organization: composition, regulation and roles of lipid rafts, Nat Rev Mol Cell Bio, 18 (2017) 361-374.

[63] M. Rappolt, M.F. Vidal, M. Kriechbaum, M. Steinhart, H. Amenitsch, S. Bernstorff, P. Laggner, Structural, dynamic and mechanical properties of POPC at low cholesterol concentration studied in pressure/temperature space, Eur Biophys J Biophy, 31 (2003) 575585.

[64] A. Hodzic, M. Rappolt, H. Amenitsch, P. Laggner, G. Pabst, Differential modulation of membrane structure and fluctuations by plant sterols and cholesterol, Biophysical journal, 94 (2008) 3935-3944.

[65] Y.C. Li, M.J. Park, S.K. Ye, C.W. Kim, Y.N. Kim, Elevated levels of cholesterol-rich lipid rafts in cancer cells are correlated with apoptosis sensitivity induced by cholesterol-depleting agents, Am J Pathol, 168 (2006) 1107-1118; quiz 1404-1105.

[66] F. Mollinedo, C. Gajate, Lipid rafts as major platforms for signaling regulation in cancer, Adv Biol Regul, 57 (2015) 130-146.

[67] S. Bose, S. Chandran, J.M. Mirocha, N. Bose, The Akt pathway in human breast cancer: a tissue-array-based analysis, Mod Pathol, 19 (2006) 238-245.

[68] K. Duvel, J.L. Yecies, S. Menon, P. Raman, A.I. Lipovsky, A.L. Souza, E. Triantafellow, Q. Ma, R. Gorski, S. Cleaver, M.G. Vander Heiden, J.P. MacKeigan, P.M. Finan, C.B. Clish, L.O. Murphy, B.D. Manning, Activation of a metabolic gene regulatory network downstream of mTOR complex 1, Mol Cell, 39 (2010) 171-183. 
[69] P. Torres-Ayuso, M. Tello-Lafoz, I. Merida, A. Avila-Flores, Diacylglycerol kinase-zeta regulates $\mathrm{mTORC1}$ and lipogenic metabolism in cancer cells through SREBP-1, Oncogenesis, 4 (2015) e164.

[70] R.S. Cantor, Lateral Pressures in Cell Membranes: A Mechanism for Modulation of Protein Function, The Journal of Physical Chemistry B, 101 (1997) 1723-1725.

[71] H. Jerabek, G. Pabst, M. Rappolt, T. Stockner, Membrane-mediated effect on ion channels induced by the anesthetic drug ketamine, J Am Chem Soc, 132 (2010) 7990-7997. [72] A. Hodzic, M. Rappolt, H. Amenitsch, P. Laggner, G. Pabst, Differential modulation of membrane structure and fluctuations by plant sterols and cholesterol, Biophys J, 94 (2008) 3935-3944.

[73] P.G. Bradford, A.B. Awad, Modulation of signal transduction in cancer cells by phytosterols, Biofactors, 36 (2010) 241-247.

[74] G.O. Ifere, A. Equan, K. Gordon, P. Nagappan, J.U. Igietseme, G.A. Ananaba, Cholesterol and phytosterols differentially regulate the expression of caveolin 1 and a downstream prostate cell growth-suppressor gene, Cancer Epidemiol, 34 (2010) 461-471.

[75] C.H. Storch, R. Ehehalt, W.E. Haefeli, J. Weiss, Localization of the human breast cancer resistance protein (BCRP/ABCG2) in lipid rafts/caveolae and modulation of its activity by cholesterol in vitro, The Journal of pharmacology and experimental therapeutics, 323 (2007) 257-264.

[76] B. Kim, H. Fatayer, A.M. Hanby, K. Horgan, S.L. Perry, E.M. Valleley, E.T. Verghese, B.J. Williams, J.L. Thorne, T.A. Hughes, Neoadjuvant chemotherapy induces expression levels of breast cancer resistance protein that predict disease-free survival in breast cancer, PloS one, 8 (2013) e62766.

[77] S. Beloribi, E. Ristorcelli, G. Breuzard, F. Silvy, J. Bertrand-Michel, E. Beraud, A. Verine, D. Lombardo, Exosomal lipids impact notch signaling and induce death of human pancreatic tumoral SOJ-6 cells, PloS one, 7 (2012) e47480.

[78] B. Kim, S. Stephen, A. Hanby, K. Horgan, S. Perry, J. Richardson, E. Roundhill, E. Valleley, E. Verghese, B. Williams, T. Hughes, J. Thorne, Chemotherapy induces Notch1dependent MRP1 up-regulation, inhibition of which sensitizes breast cancer cells to chemotherapy., BMC cancer, 15 (2015).

[79] M.E. Gonzalez, H.M. Moore, X. Li, K.A. Toy, W. Huang, M.S. Sabel, K.M. Kidwell, C.G. Kleer, EZH2 expands breast stem cells through activation of $\mathrm{NOTCH} 1$ signaling, Proceedings of the National Academy of Sciences of the United States of America, 111 (2014) 3098-3103. [80] S. Suman, T.P. Das, C. Damodaran, Silencing NOTCH signaling causes growth arrest in both breast cancer stem cells and breast cancer cells, British journal of cancer, 109 (2013) 2587-2596. 
[81] W. Zhou, G. Wang, S. Guo, Regulation of angiogenesis via Notch signaling in breast cancer and cancer stem cells, Biochimica et biophysica acta, 1836 (2013) 304-320.

[82] A.B. Awad, Y.C. Chen, C.S. Fink, T. Hennessey, beta-Sitosterol inhibits HT-29 human colon cancer cell growth and alters membrane lipids, Anticancer Res, 16 (1996) 2797-2804. [83] S. Ponnusamy, M. Meyers-Needham, C.E. Senkal, S.A. Saddoughi, D. Sentelle, S.P. Selvam, A. Salas, B. Ogretmen, Sphingolipids and cancer: ceramide and sphingosine-1phosphate in the regulation of cell death and drug resistance, Future Oncol, 6 (2010) 16031624.

[84] R.L. von Holtz, C.S. Fink, A.B. Awad, beta-Sitosterol activates the sphingomyelin cycle and induces apoptosis in LNCaP human prostate cancer cells, Nutr Cancer, 32 (1998) 8-12. [85] A.B. Awad, Y. Gan, C.S. Fink, Effect of beta-sitosterol, a plant sterol, on growth, protein phosphatase 2A, and phospholipase D in LNCaP cells, Nutr Cancer, 36 (2000) 74-78.

[86] A.B. Awad, S.L. Barta, C.S. Fink, P.G. Bradford, beta-Sitosterol enhances tamoxifen effectiveness on breast cancer cells by affecting ceramide metabolism, Mol Nutr Food Res, 52 (2008) 419-426.

[87] C. Yu, M. Alterman, R.T. Dobrowsky, Ceramide displaces cholesterol from lipid rafts and decreases the association of the cholesterol binding protein caveolin-1, J Lipid Res, 46 (2005) 1678-1691.

[88] K. Hac-Wydro, Studies on beta-sitosterol and ceramide-induced alterations in the properties of cholesterol/sphingomyelin/ganglioside monolayers, Biochim Biophys Acta, 1828 (2013) 2460-2469.

[89] P.L. Yeagle, Cholesterol and the cell membrane, Biochimica et Biophysica Acta (BBA) Reviews on Biomembranes, 822 (1985) 267-287.

[90] P.L. Yeagle, Modulation of membrane function by cholesterol, Biochimie, 73 (1991) 13031310.

[91] R. Bittman, Has nature designed the cholesterol side chain for optimal interaction with phospholipids?, Cholesterol, Springer1997, pp. 145-171.

[92] H. Ohvo-Rekilä, B. Ramstedt, P. Leppimäki, J.P. Slotte, Cholesterol interactions with phospholipids in membranes, Progress in lipid research, 41 (2002) 66-97.

[93] X. Xu, R. Bittman, G. Duportail, D. Heissler, C. Vilcheze, E. London, Effect of the structure of natural sterols and sphingolipids on the formation of ordered sphingolipid/sterol domains (rafts) Comparison of cholesterol to plant, fungal, and disease-associated sterols and comparison of sphingomyelin, cerebrosides, and ceramide, Journal of Biological Chemistry, 276 (2001) 33540-33546.

[94] K.K. Halling, J.P. Slotte, Membrane properties of plant sterols in phospholipid bilayers as determined by differential scanning calorimetry, resonance energy transfer and detergent- 
induced solubilization, Biochimica et Biophysica Acta (BBA)-Biomembranes, 1664 (2004) 161171.

[95] B. Kollmitzer, P. Heftberger, M. Rappolt, G. Pabst, Monolayer spontaneous curvature of raft-forming membrane lipids, Soft Matter, 9 (2013) 10877-10884.

[96] K. Grosjean, S. Mongrand, L. Beney, F. Simon-Plas, P. Gerbeau-Pissot, Differential Effect of Plant Lipids on Membrane Organization specificities of phytosphingolipids and phytosterols, Journal of Biological Chemistry, 290 (2015) 5810-5825.

[97] S.L. Veatch, S.L. Keller, Seeing spots: Complex phase behavior in simple membranes, Biochimica et Biophysica Acta (BBA) - Molecular Cell Research, 1746 (2005) 172-185.

[98] S.L. Veatch, K. Gawrisch, S.L. Keller, Closed-Loop Miscibility Gap and Quantitative TieLines in Ternary Membranes Containing Diphytanoyl PC, Biophysical Journal, 90 (2006) 4428-4436.

[99] T. Róg, M. Pasenkiewicz-Gierula, I. Vattulainen, M. Karttunen, Ordering effects of cholesterol and its analogues, Biochimica et Biophysica Acta (BBA)-Biomembranes, 1788 (2009) 97-121.

[100] J.A. Urbina, S. Pekerar, H.-b. Le, J. Patterson, B. Montez, E. Oldfield, Molecular order and dynamics of phosphatidylcholine bilayer membranes in the presence of cholesterol, ergosterol and lanosterol: a comparative study using 2H-, 13C-and 31P-NMR spectroscopy, Biochimica et Biophysica Acta (BBA)-Biomembranes, 1238 (1995) 163-176.

[101] A. Aufderhorst-Roberts, U. Chandra, S.D. Connell, Three-Phase Coexistence in Lipid Membranes, Biophys J, 112 (2017) 313-324.

[102] M. Kang, B. Lee, C. Leal, Three-Dimensional Microphase Separation and Synergistic Permeability in Stacked Lipid-Polymer Hybrid Membranes, Chem Mater, 29 (2017) 9120-9132. [103] P. Heftberger, B. Kollmitzer, F.A. Heberle, J. Pan, M. Rappolt, H. Amenitsch, N. Kucerka, J. Katsaras, G. Pabst, Global small-angle X-ray scattering data analysis for multilamellar vesicles: the evolution of the scattering density profile model, J Appl Crystallogr, 47 (2014) 173-180.

[104] G. Pabst, M. Rappolt, H. Amenitsch, P. Laggner, Structural information from multilamellar liposomes at full hydration: Full q-range fitting with high quality $\mathrm{x}$-ray data, Physical Review E, 62 (2000) 4000-4009.

[105] K. Grosjean, S. Mongrand, L. Beney, F. Simon-Plas, P. Gerbeau-Pissot, Differential effect of plant lipids on membrane organization: specificities of phytosphingolipids and phytosterols, The Journal of biological chemistry, 290 (2015) 5810-5825.

[106] R.F. de Almeida, E. Joly, Crystallization around solid-like nanosized docks can explain the specificity, diversity, and stability of membrane microdomains, Front Plant Sci, 5 (2014) 72. 
[107] P.A. Edwards, C. Green, Incorporation of plant sterols into membranes and its relation to sterol absorption, FEBS Letters, 20 (1972) 97-99.

[108] I. Schuler, G. Duportail, N. Glasser, P. Benveniste, M.-A. Hartmann, Soybean phosphatidylcholine vesicles containing plant sterols: a fluorescence anisotropy study, Biochimica et Biophysica Acta (BBA) - Biomembranes, 1028 (1990) 82-88.

[109] C. Rujanavech, P. Henderson, D. Silbert, Influence of sterol structure on phospholipid phase behavior as detected by parinaric acid fluorescence spectroscopy, Journal of Biological Chemistry, 261 (1986) 7204-7214.

[110] V. Swaminathan, K. Mythreye, E.T. O'Brien, A. Berchuck, G.C. Blobe, R. Superfine, Mechanical stiffness grades metastatic potential in patient tumor cells and in cancer cell lines, Cancer research, 71 (2011) 5075-5080.

[111] B.U.S.S. Chris Händel, Jürgen Schiller, Undine Dietrich, Till Möhn, Tobias R Kießling, Steve Pawlizak, AnatolW Fritsch, Lars-Christian Horn, Susanne Briest, Michael Höckel, Mareike Zink, Josef A Käs, Cell membrane softening in human breast and cervical cancer cells, New Journal of Physics, 17 (2015) 1-10.

[112] O.W. Simone Braig; B U Sebastian Schmidt; Katharina Stoiber; Chris Händel; Till Möhn, Rolf Müller, Stefan Zahler, Andreas Koeberle, Josef A Käs, and Angelika M Vollmar, Pharmacological targeting of membrane rigidity: implications oncancer cell migration and invasion, New Journal of Physics, 17 (2015).

[113] A.B. Awad, A. Downie, C.S. Fink, U. Kim, Dietary phytosterol inhibits the growth and metastasis of MDA-MB-231 human breast cancer cells grown in SCID mice, Anticancer Res, 20 (2000) 821-824.

[114] A.B. Awad, H. Williams, C.S. Fink, Phytosterols reduce in vitro metastatic ability of MDAMB-231 human breast cancer cells, Nutrition and cancer, 40 (2001) 157-164.

[115] Matczak K., Koceva-Chyla A., Gwozdzinski K., J. Z., Doxorubicin and Paclitaxel Cause Different Changes in Plasma Membrane Fluidity of MCF-7 Breast Cancer Cells, POSTĘPY BIOLOGII KOMÓRKI, 36 (2009).

[116] S. Stangl, M. Gehrmann, J. Riegger, K. Kuhs, I. Riederer, W. Sievert, K. Hube, R. Mocikat, R. Dressel, E. Kremmer, A.G. Pockley, L. Friedrich, L. Vigh, A. Skerra, G. Multhoff, Targeting membrane heat-shock protein 70 (Hsp70) on tumors by cmHsp70.1 antibody, Proc Natl Acad Sci U S A, 108 (2011) 733-738.

[117] G. Pabst, B. Boulgaropoulos, E. Gander, B.R. Sarangi, H. Amenitsch, V.A. Raghunathan, P. Laggner, Effect of ceramide on nonraft proteins, Journal of Membrane Biology, 231 (2009) 125-132.

[118] B. Boulgaropoulos, Z. Arsov, P. Laggner, G. Pabst, Stable and Unstable Lipid Domains in Ceramide-Containing Membranes, Biophysical Journal, 100 (2011) 2160-2168. 
[119] B. Boulgaropoulos, M. Rappolt, B. Sartori, H. Amenitsch, G. Pabst, Lipid sorting by ceramide and the consequences for membrane proteins, Biophys J, 102 (2012) 2031-2038. [120] T.A. Wassenaar, H.I. Ingolfsson, R.A. Bockmann, D.P. Tieleman, S.J. Marrink, Computational Lipidomics with insane: A Versatile Tool for Generating Custom Membranes for Molecular Simulations, J Chem Theory Comput, 11 (2015) 2144-2155.

[121] S.J. Marrink, H.J. Risselada, S. Yefimov, D.P. Tieleman, A.H. de Vries, The MARTINI force field: Coarse grained model for biomolecular simulations, Journal of Physical Chemistry B, 111 (2007) 7812-7824.

[122] B.N. Olsen, P.H. Schlesinger, D.S. Ory, N.A. Baker, 25-Hydroxycholesterol increases the availability of cholesterol in phospholipid membranes, Biophys J, 100 (2011) 948-956.

[123] S.A. Hutchinson, D.A. Kane, H. Røberg-Larsen, K. Vaghela, J.L. Thorne, Cholesterol hydroxylation products confer resistance to anthracyclines in Triple Negative Breast Cancer, Proceedings of the Nutrition Society, 77 (2018) E1.

[124] M.E. Sherman, J.D. Figueroa, J.E. Henry, S.E. Clare, C. Rufenbarger, A.M. Storniolo, The Susan G. Komen for the Cure Tissue Bank at the IU Simon Cancer Center: A Unique Resource for Defining the "Molecular Histology" of the Breast, Cancer prevention research, 5 (2012) 528-535. 